\title{
Art et langage chez Ernst Cassirer : morphologie et/ou structuralisme?
}

Muriel van Vliet

\section{(2) OpenEdition}

1 Journals

Édition électronique

URL : http://journals.openedition.org/imagesrevues/3496

DOI : 10.4000/imagesrevues.3496

ISSN : $1778-3801$

Éditeur :

Centre d'Histoire et Théorie des Arts, Groupe d'Anthropologie Historique de l'Occident Médiéval, Laboratoire d'Anthropologie Sociale, UMR 8210 Anthropologie et Histoire des Mondes Antiques

\section{Référence électronique}

Muriel van Vliet, " Art et langage chez Ernst Cassirer : morphologie et/ou structuralisme ? », Images Re-vues [En ligne], Hors-série 5 | 2016, mis en ligne le 01 janvier 2014, consulté le 30 janvier 2021. URL : http://journals.openedition.org/imagesrevues/3496 ; DOI : https://doi.org/10.4000/ imagesrevues.3496

Ce document a été généré automatiquement le 30 janvier 2021.

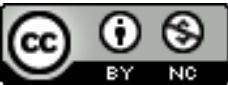

Images Re-vues est mise à disposition selon les termes de la Licence Creative Commons Attribution Pas d'Utilisation Commerciale 4.0 International. 


\section{Art et langage chez Ernst Cassirer : morphologie et/ou structuralisme?}

Muriel van Vliet 


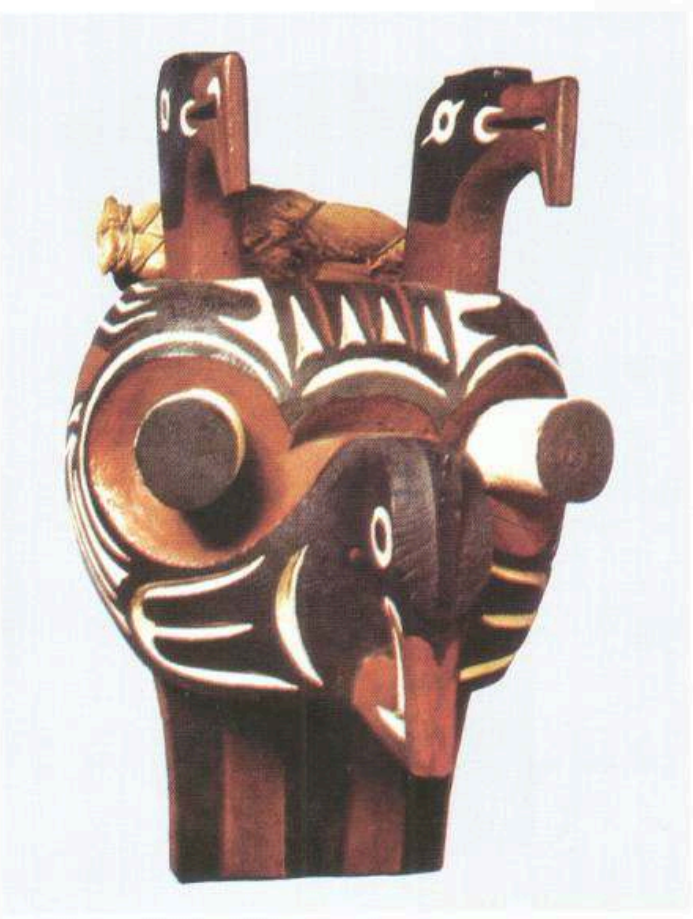

Masque swaihwé (Cowichan). New York, Museum of the American Indian, Heye Foundation. Exposé dans Claude Lévi-Strauss, La voie des masques, Paris, Plon, 1979, cahier central d'images, illustration 5.

\section{Introduction}

1 Dans la philosophie de la culture que dessine progressivement Cassirer selon le jeu combinatoire $^{1}$ des différentes formes symboliques qui en constituent les lignes de force, l'art et le langage sont toujours étudiés conjointement, sans être toutefois purement et simplement identifiés, par contraste avec l'approche que développe Benedetto Croce de manière contemporaine en Italie, finissant par réduire quant à lui, pour prolonger Hegel, la théorie du langage à l'esthétique et vice et versa ${ }^{2}$. S'il est vrai que l'art ne peut être approfondi sans une approche langagière et que le langage ne peut réciproquement pas être compris dans toute sa profondeur sans une approche artistique de la culture, il ne s'agit jamais de réduire l'art au langage ou le langage à l'art chez Cassirer. Afin de mieux saisir la complexité de leur " jeu », au double sens de leur interaction et de leur écart, il convient de bien dissocier plusieurs moments de son analyse. Ceux-ci ne correspondent pas tant à des étapes chronologiques de l'évolution de Cassirer qu'à l'histoire de l'exégèse de son œuvre par ses interprètes successifs. Ils correspondent à plusieurs niveaux selon lesquels, à juste titre, le rapport de l'art au langage peut être diversement éclairé chez cet auteur.

2 À un premier niveau, il est bien connu que Cassirer a joué avec le concept de forme symbolique un rôle important pour comprendre le tournant langagier que Panofsky fait subir à l'histoire de l'art avec l'élaboration de la méthode iconologique. Dans ce cadre, plusieurs questions se posent : comment Cassirer a-t-il pu combiner sans contradiction 
la forme a priori kantienne et les figures de l'esprit hégéliennes pour élaborer cette notion de forme symbolique ? Quel rôle le théoricien du langage Wilhelm von Humboldt a-t-il joué en amont en faisant opérer un tournant langagier au criticisme kantien? Comment Panofsky a-t-il élaboré la méthode iconologique d'interprétation des œuvres d'art à partir de la notion de forme symbolique et en prenant indirectement, via Cassirer, appui sur Humboldt ? Se distancie-t-il en cela de Cassirer? Certaines de ces questions ont été abordées au plan de la littérature de recherche, notamment par Sylvia Ferretti ${ }^{3}$ et par Michael Ann Holly ${ }^{4}$; le récent ouvrage d'Audrey Rieber contribuant à remettre en perspective ces approches et à les discuter ${ }^{5}$.

3 Toutefois, à un second niveau d'approche, on a cherché à interroger Cassirer à la lumière de deux autres univers de pensée: d'une part, en amont, à celle de la morphologie goethéenne et, d'autre part, en aval, à celle du structuralisme de Claude Lévi-Strauss. Selon cette perspective, il ne s'agit alors plus tant de situer Cassirer "entre» Kant et Hegel, qu' "entre» Goethe et Lévi-Strauss. Cela a été notamment l'approche plus ou moins implicite de John Michael Krois, co-directeur récemment disparu du centre sur l'image « Bildakt und Verköperung » de Berlin ${ }^{6}$. De 1933 à 1945, Cassirer prend en effet acte d'un nouveau tournant langagier dans les sciences humaines, renforçant le tournant humboldtien imprimé au kantisme : il s'agit bien sûr du tournant structuraliste, que Cassirer est l'un des premiers, voire le premier à dénommer et à prendre en compte comme tel à titre de courant global de pensée, caractéristique du début des années $40^{7}$. Selon lui, le structuralisme est illustré non seulement par la linguistique structurale de Jakobson dont il approfondit les vues sur le bateau de l'exil qui les conduit tous deux de Suède jusqu'aux États-Unis et au sein du Cercle Linguistique de New York, mais aussi par la psychologie de la Gestalt telle que l'exploite Kurt Goldstein, son cousin neurobiologiste dont il connaît les enquêtes sur les pathologies de la conscience symboliques pour être allé visiter à plusieurs reprises les hôpitaux et centres d'études où il travaillait avec, notamment, Adhémar Gelb, et par la science de l'art élaborée par Wölfflin, dont les concepts fondamentaux pour penser l'histoire de l'art fonctionnent déjà comme autant d'invariants structuraux. Dans cette démarche, Cassirer enracine directement ce «retour à la forme » dont témoigne le structuralisme naissant à la fois sur la morphologie goethéenne et sur la théorie du langage de W. von Humboldt. Le structuralisme aurait-il alors ses racines profondes en Allemagne? C'est précisément ce que les écrits de Cassirer sur le structuralisme invitent à réévaluer.

4 Néanmoins, si la notion de forme symbolique peut être avec pertinence lue encore comme Gestalt (au sens où l'entend Goethe) et déjà comme structure (au sens que lui donne Lévi-Strauss), et s'il peut sembler pertinent de rapprocher de ce point de vue Cassirer de Lévi-Strauss ${ }^{8}$, il demeure des points de divergence essentiels entre leurs approches « sémiologiques » respectives de la culture ${ }^{9}$. À la différence de Lévi-Strauss, Cassirer ne néglige jamais le corps et son espace affectif de projection dans l'approche des phénomènes culturels et il accorde, comme l'historien de l'art que fut Aby Warburg, un primat explicite au rituel sur le mythe, ce qui a des répercussions directes sur la compréhension du rapport de l'image au texte et du visuel au langage ${ }^{10}$. «Am Anfang war die Tat »: "au commencement était l'action», et non le mot, non la pensée discursive et représentationnelle ${ }^{11}$. Il faut admettre selon Cassirer un exercice actif de la fonction symbolique dès le niveau de la perception sensible et, a fortiori, dès l'organisation d'un espace affectif par le rituel et par la création artistique, qui contribuent à une orientation originaire décisive pour tout le processus de 
construction du rapport moi-monde, y compris selon ses dimensions cognitive et théorique. C'est donc sans doute la proximité entre Warburg et Cassirer ${ }^{12}$ qu'il faut sur ce point en définitive retenir pour cerner leur approche contrastée de ce que j'appellerai, avec les anthropologues Michael Houseman et Carlo Severi, la "symbolisation rituelle ${ }^{13}$. Celle-ci régit non seulement la pensée mythique, mais aussi, d'une autre manière, l'art: l'histoire de l'art s'ouvre alors non seulement sur la théorie du langage, mais aussi sur l'ethnologie et l'anthropologie de l'image. Définir une grammaire des affects devient le cœur d'un projet commun consistant à élaborer ce que Warburg désigne dans une lettre à Cassirer comme une "science universelle " de la culture comme anthropologie « de l'homme en mouvement » ${ }^{14}$. S'il est fructueux d'interpréter la philosophie des formes symboliques comme une anthropologie structurale «avant la lettre $»^{15}$, force est de constater que Cassirer propose une version du structuralisme nettement distincte de cette que Lévi-Strauss développera: une conception plus profondément et plus explicitement «morphologique» que celle de l'anthropologue français, sans doute à même d'éviter certains écueils « formalistes » ou « intellectualistes », que rencontre ce dernier, indubitablement, dans certains textes et qui seront d'ailleurs source de critiques menées à son égard.

Notre article propose d'approfondir ces trois niveaux, pour mieux cerner l'ancrage du structuralisme cassirerien dans la morphologie et l'importance de cet ancrage : revenir d'abord sur le rapport de Cassirer à Panofsky et la nature de ce que l'on pourrait nommer le "premier » tournant langagier, d'inspiration humboldtienne, permettant d'éclairer l'adoption par Panofsky d'une perspective iconologique sur l'art, ouvrant sur " une vision totale » jetée sur chaque culture déterminée, par contraste et contre-point avec d'autres ; ensuite, se concentrer sur l'inflexion des positions de Cassirer avec l'avènement de la linguistique structurale (Jakobson), qui correspondrait alors à un "second» tournant langagier approfondissant le premier; et, enfin, questionner l'élaboration d'une "science universelle de la culture" comme anthropologie de "l'homme en mouvement", permettant d'aborder le processus de signification sous l'angle d'une "symbolisation rituelle", dont la pensée mythique comme l'art témoignent diversement. Sans doute ce kaléidoscope peut-il être un prisme intéressant pour aborder les problématiques actuelles qui s'ouvrent lorsque l'on chercher à « faire retour au langage » (et même au texte et à sa discursivité) pour aborder le champ de l'esthétique, élargi du côté de la théorie du langage et de l'anthropologie.

\section{De la forme a priori kantienne et des figures de l'esprit hégéliennes à la forme symbolique : l'importance de Humboldt (via Cassirer) pour comprendre l'iconologie de Panofsky}

6 Bien que cela ait été déjà étudié, il n'est pas inintéressant de chercher à comprendre le rôle de Cassirer dans l'élaboration de l'iconologie de Panofsky, car celui joué par Humboldt y est souvent négligé, ce qui provoque une série de mécompréhensions quant au véritable sens de la démarche iconologique. Dans cette perspective, il faut suivre ce qui peut sembler à première vue un détour par la théorie du langage du père de la linguistique comparée que fut W. von Humboldt.

Il s'agit de bien comprendre ce que Fabien Capeillères a nommé «l'historicisation du transcendantal kantien » chez Cassirer et Panofsky ${ }^{16}$. Cassirer a longtemps été abordé 
comme un membre de l'École de Marbourg, c'est-à-dire comme un épistémologue néokantien, proche d'Hermann Cohen et de Paul Natorp ${ }^{17}$. Cette école a, comme on le sait, tenté de "revenir à Kant ", selon le mot d'ordre d'É. Zeller, «pardelà Hegel »: elle a tenté de pallier aux défauts du criticisme à la lumière de la phénoménologie hégélienne, tout en affirmant ne faire finalement que poursuivre le projet kantien en l'élargissant, de la critique de la raison à celle de la culture.

Selon Cassirer, il faut de fait rénover la forme a priori kantienne en lui imprimant deux caractéristiques propres aux figures de l'esprit hégélienne. Il faut en effet reconnaitre à la forme ses dimensions historique et langagière intrinsèques. Le langage n'est pas un auxiliaire contingent de la pensée, il en est le « médium » indispensable et c'est dans le «milieu » du langage que se constitue diversement le rapport moi-monde ${ }^{18}$. On ne peut, comme l'a fait Kant, reléguer l'étude du langage dans un " appendice » du système ${ }^{19}$ et ne l'aborder que sous son angle empirique ou pragmatique. Son statut est en effet « constitutif » ou, pour employer le vocabulaire kantien, «transcendantal».

8 En quel sens toutefois la conception phénoménologique hégélienne doit-elle tout autant être modifiée? Pour critiquer Hegel, Cassirer se positionne dans le sillage de l'adversaire que fut historiquement, vis-à-vis de Hegel - qui le mentionne d'ailleurs dans ses écrits - Wilhelm von Humboldt. Ce théoricien du langage a, selon Cassirer, fait subir au criticisme un tournant langagier décisif ${ }^{20}$ présentant l'avantage de ne pas refermer, comme Hegel a pu le faire, l'approche du langage exclusivement sur «le logique »: l'approche humboldtienne permet une véritable sémiologie ${ }^{21}$. La présentation téléologique des figures de l'esprit hiérarchise chez Hegel toutes les formes de la culture et réduit, si l'on peut dire, la fonction de sens que chacune a pu apporter au seul profit du «logique ", principe unique absolu. Or, Cassirer reproche précisément à Hegel

Pour Humboldt comme pour Cassirer, trois caractéristiques du langage doivent être mises en lumière : sa dimension non seulement représentative mais aussi expressive, et sa dimension dialogique. Le langage est pour Humboldt à la fois energeia et ergon. Dans chaque acte de parole, le langage comme "parole parlée » est recréé comme "parole parlante " (ce que reprendra Merleau-Ponty à Humboldt pour sa propre théorie de l'expression via sa lecture assidue de Cassirer). Cassirer veut appliquer le tournant langagier déjà imprimé au criticisme kantien par Humboldt à tout le champ de la culture, c'est-à-dire à l'étude des mythes, de l'art, des sciences et des techniques. En s'appuyant sur Humboldt, les différentes fonctions de sens que Cassirer met au jour à travers l'étude du langage dans le premier tome de la Philosophie des formes symboliques (1923) sont transposées progressivement à l'étude de la pensée mythique et de l'art $(1925)^{28}$. Dans ces deux domaines, c'est en premier lieu la fonction de sens qu'est l'expression qu'il faut mettre au jour, « expression » traduisant l'allemand « Ausdruck ». L'expression correspond à la construction active d'un espace de vie, de pensée et d'action où l'homme fait l'expérience vivante de sa liberté2 ${ }^{9}$.

11 Dès le niveau de la perception sensible la plus fruste se construit un «entrelacement " ("Verflechtung») du sensible et du sens: chaque donné qui se présente "ici » et " maintenant » à moi est aussitôt inséré dans une expérience globale où elle prend sens en fonction du principe de la mise en série par laquelle on construit ce donné, et ce, en fonction d'une certaine visée ${ }^{30}$. Le rapport du donné à l'expérience globale est 
comparable au rapport mathématique du différentiel à l'intégrale. La fonction symbolique s'exerce avant même la production explicite et consciente de signes et de symboles.

12 L'expression des émotions dans des mimiques, recourant à un langage gestuel, ou dans des danses rituelles, par exemple, relève de ce niveau. Dans ces phénomènes de danse ou de gestes expressifs, les affects sont activement réélaborés. Ils ne peuvent pas être simplement considérés d'un point de vue biologique (naturaliste) comme chez Darwin ${ }^{31}$, mais doivent l'être du point de vue de la théorie du langage, de l'esthétique et de l'étude de la pensée mythique - à un niveau sémiologique. L'élaboration humaine d'une grammaire des affects permet une première distance entre le moi et le monde et donc une première forme de liberté, avec une différence au moins de degré, sinon de nature, avec le monde animal: seul l'homme travaille dès le niveau de l'expression des affects selon la modalité du possible. On ne peut aborder les symboles humains de manière déterministe sans les amputer d'une partie de leur valeur.

Le mouvement mimique est «l'identité immédiate de l'intérieur' et de l"extérieur', du 'spirituel' et du corporel', dans la mesure précisément où ce qu'il est directement et de façon sensible signifie et 'dit' quelque chose, mais de présent en lui ${ }^{32}$, Cassirer utilisant pour qualifier ce mouvement mimique la métaphore de "l'insoluble entrelacement " ${ }^{33}$ $\mathrm{du}$ sensible et du sens. Cette thèse, selon laquelle l'identité du sensible et du sens doit être cherchée et trouvée dans le milieu que constitue le langage et selon laquelle celui-ci comporte une phase mimique décisive, est déjà présente chez W. von Humboldt. Cassirer l'explicite : «Il ne s'agit pas ici d'un simple passage, d'une addition arbitraire du signe mimique à l'état émotif qu'il désigne, mais les deux éléments, l'émotion et son extériorisation, la tension intérieure et sa libération, sont donnés en un seul acte temporellement indivisible $»^{34}$.

14 Alors que, selon Darwin, «l'expression d'une émotion ne serait rien d'autre que la forme atténuée d'un ancien acte concret finalisé; l'expression de la colère, par exemple, l'image affaiblie et pâlie d'un ancien mouvement d'agression, celle de la frayeur, l'image d'un mouvement de défense, etc. »" ${ }^{35}$, selon Cassirer, «tout mouvement d'expression élémentaire constitue de fait un premier seuil du développement spirituel dans la mesure où il est encore entièrement situé dans l'immédiateté de la vie sensible et par ailleurs en est déjà sorti $\aleph^{36}$. Ici, on peut remarquer que Cassirer transpose au champ de la vie affective la dialectique hégélienne animant le premier chapitre sur la certitude sensible de la Phénoménologie de l'esprit ${ }^{37}$. Il fait "régresser d'un cran", pourrait-on dire, l'analyse hégélienne, en repérant la spontanéité de l'esprit dès le niveau de l'expression des émotions, revalorisant davantage que Hegel l'aisthesis et attirant de ce fait l'attention sur l'importance du schéma corporel.

Au niveau de l'art, joue également une autre fonction de sens mise au jour dans l'étude du langage, à savoir la dimension présentative $e^{38}$. La "présentation" traduit ici l'allemand Darstellung ${ }^{39}$. Alors que dans le rituel de danse de la pensée mythique, le danseur est identifié au dieu, ou l'épi de maïs du cérémoniel magique littéralement identifié au cosmos, dans la danse artistique, les spectateurs et acteurs ont au contraire pleinement conscience du jeu des danseurs comme jeu : alors que l'expression de la pensée mythique témoigne d'un phénomène de concrescence ou de coalescence du signifiant et du signifié, ces deux pôles n'étant, pour le dire plus exactement, pas encore distingués (ce qui fait d'ailleurs toute la force de ce mode d'expression), la présentation artistique témoigne au contraire de la faculté de distinction et 
d'articulation du signifiant et du signifié. Lorsque l'artiste reprend des formes du mythe qui insistent en lui du fait de la forte polarisation affective dont le mythe est porteur, il les «libère pour l'humanité $»^{40}$. L'art met fin à la substitution, propre à la dialectique de la pensée mythique, d'un déterminisme naturel au profit d'un déterminisme plus redoutable encore : le déterminisme « culturel ». La tragédie propre à la pensée mythique consiste en effet à destituer l'homme de la liberté dont la production de mythes et de rituels était pourtant la première marque : l'homme de la pensée mythique finit par être plus terrifié par le mot tabou que par un danger naturel véritable. Or, devant une œuvre d'art, nous ne frémissons plus comme devant l'idole et en entendant les poèmes épiphaniques, nous ne tremblons plus comme en entendant le mot tabou. L'image est reconnue « en tant que telle ${ }^{41}$. L'art articule les deux fonctions de sens que sont l'expression et la présentation et gagne précisément de l'intensité en oscillant sans cesse entre présentification expressive et présentation distanciatrice, c'est-à-dire entre l'Ergreiffen (la saisie à pleines mains de ce qui menace - le rituel de la pensée magique) et le Begriff (le concept, qui met cette menace à distance - la science) ${ }^{42}$.

Pour le dire autrement, le mythe et le langage apparaissent imbriqués quand on met au jour leur racine commune dans le phénomène de ce que Cassirer nomme la "métaphore originaire", en allemand: l'Urmetapher. Le long essai intitulé Sprache und Mythos, sous-titré "Ein Beitrag zum Problem der Götternamen » ${ }^{43}$ est particulièrement intéressant sur ce point pour comprendre l'imbrication de l'art et du mythe. Une pensée qui fonctionne selon le principe de la métaphore originaire est élaborée selon le principe " pars pro toto ", la partie étant prise littéralement pour le tout. Pour reprendre l'exemple précédent: l'épi de maïs du cérémoniel des Indiens Cora au NouveauMexique n'est certes en réalité qu'un élément de la nature. Or, il est, dans le rituel, identifié au tout de l'univers : selon une pensée qui fonctionne encore exclusivement selon le principe de la métaphore originaire, il ne représente pas le cosmos, il est le $\operatorname{cosmos}^{44}$. Le langage et le mythe ne se séparent que quand le langage accède à la fonction de sens qu'est la présentation, ce qui correspond à la séparation originaire qui donne naissance à la religion et à l'art ${ }^{45}$ (" séparation originaire » traduisant Urteilung, avec un jeu de mots intraduisible en français, le mot oscillant entre l'idée de "séparer » (teilen) et celle de «jugement» $\left.(\text { Urteil })^{46}\right)$. Dans une métaphore poétique, la partie reste certes identifiée au tout, mais le lecteur a conscience du « comme si ». Le signifiant et le signifié ne sont pas tout bonnement identifiés l'un à l'autre. La métaphore poétique serait justement, selon Cassirer, un résidu de cette racine originaire ${ }^{47}$ du langage et du mythe, c'est-à-dire d'une forme de pensée "sauvage » (et créative) qui se conserve au plan fonctionnel, malgré l'abandon de la croyance accordée au contenu de la pensée mythique et à laquelle nous pouvons continuer de puiser, ainsi que le font les poètes.

À un autre niveau, Cassirer souligne l'importance chez W. von Humboldt de la notion de "vision du monde» (Weltansicht) introduite par sa théorie du langage. Chaque langue contribue à construire activement le rapport moi/monde. Or, les langues sont diverses. Il faut alors admettre une pluralité irréductible des «manières de faire des mondes", par homologie structurale avec la pluralité irréductible des langues ${ }^{48}$. Ce pluralisme des universaux permet de dépasser aussi bien la conception anhistorique de la forme proposée par Kant que la conception téléologique des figures de l'esprit propre à Hegel. Il ne s'agit plus d'ordonner téléologiquement les formes symboliques, mais plutôt d'établir leur jeu combinatoire sous la forme d'un réseau ouvert ${ }^{49}$. Le mythe ou l'art ne sont pas «inférieurs " à la science, bien qu'elle soit la seule à accéder à la fonction de sens qu'est la «signification pure » (reine Bedeutung, utilisation de symboles 
dont le sens dépend d'un système choisi, comme la table des éléments de N. Bohr). Ils servent de complément, de compensation ${ }^{50}$ ou de contrepoint nécessaires à l'attitude scientifique et technologique, en permettant de faire halte au seuil du processus de construction globale du sens. Il faut souligner que la notion de «vision du monde » ne met aux yeux de Humboldt ou de Cassirer aucunement la philosophie en position maitresse (et encore moins le «logique»). Chaque forme symbolique, qu'il s'agisse du langage, de l'art, de la technique, de la science, de l'histoire - selon une liste que Cassirer présente d'ailleurs toujours comme explicitement non-exhaustive - présente une rationalité spécifique, cette rationalité pouvant très bien se construire de manière non discursive.

Comment comprendre dès lors le recours de Panofsky à la forme symbolique cassirerienne dans son iconologie? En quoi sa méthode est-elle éclairée par les différents points de la pensée de Cassirer que nous venons de souligner?

Fig. 2.

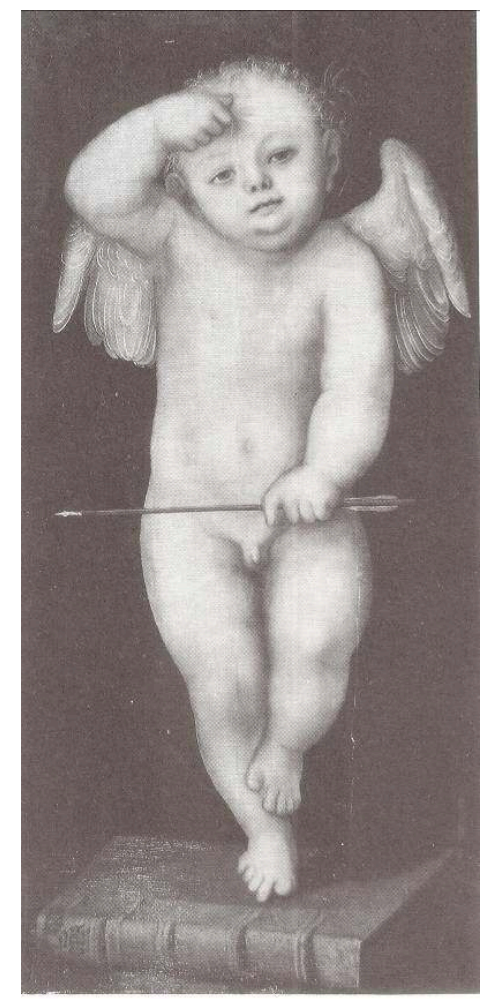

Lucas Cranach l'Ancien, Cupidon ôte son bandeau. Commenté par Erwin Panofsky, Essais d'iconologie, Les thèmes humanistes dans l'art de la Renaissance, illustration 106, Paris, Gallimard, 1967, p. 202.

Le premier niveau, pré-iconographique, mis au jour par Panofsky, correspond à la signification immédiate, factuelle et expressive ${ }^{51}$. On peut remarquer que Panofsky dit qu'elle est déjà activement le produit d'une culture, bien qu'elle ne nécessite que le bagage culturel le plus fruste. Par exemple, pour indiquer la signification factuelle et expressive immédiate du petit Éros de Cranach L'Ancien représenté dans Cupidon ôte son bandeau $^{52}$ (fig. 2), on peut dire qu'il s'agit d'un petit enfant, en équilibre sur un livre, qui semble à première vue un peu triste. Au niveau de l'expression (Ausdruck), il y a déjà création active d'un espace de vie et de pensée, mais il faut bien reconnaître que dans le développement de ses analyses iconologique, cette couche de sens, à laquelle Cassirer 
consacre de nombreuses analyses, n'est que "survolée" par Panofsky ${ }^{53}$. Ce qui l'intéresse davantage est en effet le niveau de la signification conventionnelle, le niveau iconographique où l'on peut mettre en parallèle des textes et des images pour chercher ce qu'elles «présentent». Dans l'exemple du tableau de Cranach, la convention veut que l'on représente Éros sous la forme d'un enfant ailé, portant un arc. On peut, pour restituer le sens de ce tableau, retracer toute l'histoire du thème de l'amour aux yeux bandés à travers divers textes, ce à quoi s'emploie Panofsky. Le niveau de la Darstellung intéresse Panofsky davantage que celui de l'Ausdruck. Il ne se préoccupe que très peu de l'image comme lieu d'élaboration d'un espace orienté par notre schéma corporel et par la polarité de nos affects, mais la considère plutôt comme un analogon aux textes avec laquelle ils constituent une même épistémè ${ }^{54}$, traduisant des «forces formatrices d'habitudes» similaires ${ }^{55}$. Cependant, il faut souligner que Panofsky ne réduit pas l'image au texte, car un troisième niveau essentiel vient corriger et intégrer, voire même reconfigurer les niveaux précédents : il s'agit de la signification symbolique, le niveau iconologique au sens strict, analysant l'image comme le "symptôme " ${ }^{56}$ d'une certaine "vision du monde» (Weltansicht) ou "vision totale " (gesamte Sicht). Or, cette vision du monde comprend non seulement le visuel, mais renvoie surtout à un tout un système culturel complexe (incluant religion, économie, techniques, sciences...). Pour poursuivre notre exemple, il faut remarquer que le petit Éros de Cranach est en équilibre sur un ouvrage de Platon: c'est tout le monde néo-humaniste florentin que cette image "trahit ", selon Panofsky. À ce troisième et dernier niveau, l'iconologie ouvre l'histoire de l'art sur la philosophie, sur la psychologie, sur l'étude des religions, sur la sociologie et sur les problèmes posés successivement par la théorie de la connaissance en général. Pour donner un exemple plus ample encore, l'ouvrage de Panofsky sur La perspective comme forme symbolique $e^{57}$ ne réduit pas les tableaux à des textes figés dont ils seraient simplement l'allégorie, mais interroge plus profondément la construction symbolique de l'espace et son évolution en fonction d'épistémai rétrospectivement bien distinctes. Il n'est dès lors pas étonnant que Pierre Bourdieu ait pu s'inspirer de la notion d'habitus culturel développée déjà par Panofsky pour ses propres réflexions sur l'organisation sociologique, notamment ses réflexions sur l'organisation spatiale de l'habitat kabyle ${ }^{58}$.

Nous avons montré, dans un premier temps, la corrélation que l'on peut établir, avec des déplacements d'accent, entre les fonctions de sens que l'analyse cassirerienne du langage a mis au jour (Ausdruck, Darstellung, et le niveau de la «vision totale » où l'on accède à la "vision du monde " (Weltansicht) propre à une culture particulière) et la conception que se fait Panofsky de l'iconologie. Nous avons pour cela suivi le chemin cassirerien qui mène de la forme a priori kantienne et des figures de l'esprit hégéliennes à l'iconologie de Panofsky, en passant par l'analyse du tournant langagier qu'Humboldt déjà avait proposé de faire subir au criticisme. Mais voyons maintenant comment ce tournant langagier s'infléchit avec l'avènement du structuralisme et la prise de conscience par Cassirer de l'importance de la méthode développée par Jakobson pour l'ensemble de ce qu'il nomme encore "sciences de la culture », mais qui deviendront bientôt, à partir de 1945, « sciences humaines ». 


\section{Un second " tournant langagier » : de la Gestalt (Goethe) à la structure (Lévi-Strauss)}

21 Pour bien comprendre l'œuvre de Cassirer, le pari qu'a fait John M. Krois a été de souligner non pas tant la manière dont il parvient à combiner sans contradiction, en s'appuyant sur W. von Humboldt, le criticisme kantien et la phénoménologie hégélienne, mais plutôt l'importance du point de départ et celle du point d'arrivée de son parcours ${ }^{59}$. Le point de départ des études cassireriennes n'est pas en effet pas l'étude des philosophies de Kant ou de Hegel, mais bien celle de l'œuvre de Goethe, en tant que botaniste et en tant que poète. Et le point d'arrivée de son cheminement intellectuel, est marqué, en 1945, par son article sur le structuralisme dans la linguistique contemporaine paru dans la revue Word, une revue née dans le Cercle Linguistique de New York, à laquelle collaborent Cassirer, Jakobson et Lévi-Strauss. Ce n'est qu'ainsi, et non pas en partant de Kant et de Hegel, que l'on peut saisir le rôle charnière qu'a pu jouer la notion de forme symbolique, permettant de passer, avec certains aménagements, de la notion goethéenne de Gestalt à la notion lévi-straussienne de structure. Et cela, si l'on en suit les analyses de Cassirer, apparemment sans véritable solution de continuité, car il ancre le structuralisme naissant sur une morphologie goethéenne et une théorie du langage humboldtienne reconfigurées par ses soins. Du point de vue de ce second tournant, c'est bien sûr encore une nouvelle conception du rapport art/langage qui se dessine chez Cassirer.

Recommençons donc par le véritable point de commencement, c'est-à-dire par l'approche cassirerienne de l'œuvre de Goethe ${ }^{60}$. Cassirer n'a nullement entamé ses études par des cours de philosophie, mais par un ensemble de cours sur le droit (comme le voulait sa famille) et sur la littérature (suivant en cela son propre penchant). Il a eu, à Heidelberg et Berlin, les meilleurs professeurs spécialistes de Goethe de son époque, entre autres les professeurs Kuno Fischer (sur Faust) et Eric Schmidt. La philologie goethéenne était alors en plein essor. Mais les spécialistes de philologie cherchaient surtout à identifier les personnages des romans, essais et pièces de théâtre de Goethe avec des personnages de sa vie réelle, suivant un courant que l'on peut rétrospectivement qualifier de "biographisme». William Scherer est par exemple persuadé que dans Le Satyre ou le Diable des bois divinisé, le Diable n'est autre qu'Herder. E. Schmidt pense que Méphisto est Merk, tout comme dans Richardson, Rousseau et Goethe, Schmidt prétend «savoir» qui «sont» en réalité les figures principales de la Nouvelle Héloïse. Or, Cassirer prend d'emblée le contre-pied de cette approche caractérisée par un biographisme réducteur. Il veut surtout ne pas couper la partie poétique de la partie de son œuvre consacrée à la botanique. Il refuse par ailleurs de "sectionner» son œuvre, en y découpant artificiellement des périodes aux caractéristiques opposées ${ }^{61}$. Enfin, il veut reconnaître en lui un philosophe, comme en témoigne le premier des 23 essais qu'il consacra à cet auteur, intitulé "Goethe et Platon" en $1920^{62}$. Il souhaite laisser Goethe s'interpréter par lui-même, sans faire intervenir d'élément extérieur qui conditionnerait son projet d'écriture. Il propose d'en faire une lecture morphologique ou structuraliste " avant la lettre ». Il ne s'agit alors ni de morceler l'œuvre en périodes, ni de séparer l'œuvre et la vie de Goethe, mais bien de travailler sur le fondement d'homologies structurales complexes entre la vie et l'œuvre d'une part et entre les œuvres elles-mêmes d'autres part, en reconstruisant l'ensemble autour de quelques problèmes centraux, des "formes internes", qui se définissent 
comme autant de principes de mise en série des œuvres et de la vie de Goethe. Citons Cassirer plus en détail : « Pour pénétrer jusqu'aux racines proprement dites de la forme goethéenne, il n'est par conséquent pas besoin de tenir compte des détails biographiques, ni du cours extérieur de l'existence ; ils devront être inclus dans les pures conditions de la création elle-même et être compris à partir de ces conditions. Ce rapport fondamental ne 'devient' pas propre à Goethe, il l'est dès le premier moment où celui-ci vient à se produire. La voie qu'il suit ne vise pas à mettre un terme à une aspiration sans limite, mais conduit vers une totalité qui possède en elle-même sa mesure et ses frontières internes $»^{63}$. On trouve ici déjà exprimée l'exigence de n'expliquer les mythes qu'à partir d'eux-mêmes et de ne faire intervenir les données de l'infrastructure que de manière annexe et secondaire, telle que C. Lévi-Strauss l'exprimera: "On prétend expliquer des types d'ordres en les ramenant à des contenus qui ne sont pas de même nature, et qui, par l'effet d'une contradiction singulière, agiraient sur leur forme du dehors. Le structuralisme authentique cherche, au contraire, à saisir avant tout les propriétés intrinsèques de certains types d'ordre. Ces propriétés n'expriment rien qui leur soit extérieur $»^{64}$.

Si Cassirer s'oppose à une lecture qui rabattrait les œuvres sur le contenu historique contingent en faisant de l'œuvre leur reflet, cela ne signifie aucunement qu'il ne va pas lui aussi orienter sa lecture en montrant un développement du corpus des œuvres, mais cela signifie qu'il cherche leur loi de développement interne dans un rapport d'homologie structurale avec le développement de la forme de la vie de Goethe et le développement de la forme de son œuvre scientifique. La diachronie n'est pas exclue par l'approche morphologique, elle lui est intrinsèque, mais elle ne peut être envisagée comme une juxtaposition extérieure de faits. Diachronie et synchronie sont deux faces du même processus d'analyse du développement progressif de l'ensemble d'une œuvre et de l'histoire de leur auteur en fonction d'un principe dynamique d'orientation de la série des œuvres et des événements de la vie. Ce principe n'est pas un concept abstrait qui réduirait la spécificité de chaque moment. Ce n'est pas le modèle hégélien de la fleur qui est «négation du bouton » qui est ici convoqué, -Cassirer précise que Goethe abhorrait ce modèle malgré une certaine amitié pour Hegel et l'influence qu'il a sans doute exercée sur son œuvre-, mais celui de la transformation continue de l'organique (ce que Cassirer pense être un apport leibnizien chez Goethe). Des rapports entre éléments constants présents du début à la fin du processus évoluent progressivement jusqu'à leur maturation, si bien que les premières œuvres sont éclairées en retour par la nouveauté qui émerge des œuvres postérieures. Il ne s'agit pas d'opposer le Goethe du Sturm und Drang au Goethe de l'époque classique, mais de voir comment un même problème parcourt intensément et de manière dynamique toute son œuvre, ce qui n'exclut pas des périodes d'opposition, d'inversion, bien au contraire, mais ces oppositions ne font sens que quand elles sont intégrées dans une même unité dynamique $^{65}$. C'est pourquoi Cassirer essaie de montrer comment, dès les œuvres de jeunesse, sont présents des éléments que l'on retrouvera accentués dans les œuvres de vieillesses (par exemple le motif du renoncement), tandis que l'intensité des œuvres de jeunesse se verra non pas amoindrie, mais tout simplement "re-polarisée » différemment dans les œuvres de vieillesse. Cassirer complexifie une approche linéaire du temps de la création en montrant que justement, toute nouvelle création est aussi recréation des œuvres précédentes. Il ne s'agit pas de mentionner linéairement telle œuvre puis tel événement dans la vie de Goethe, puis telle œuvre, puis tel événement de la vie, etc. de manière plate; mais de faire des mouvements de va-et-vient dans le cours 
du temps de la création pour montrer combien tout se tient malgré les tensions réelles entre divers éléments sans cesse dépolarisés et repolarisés autour de cas saillants, pour reprendre à dessein le vocabulaire d'Aby Warburg.

Or, par une sorte de mise en abîme, l'un des problèmes identifié par Cassirer comme principe interne du développement de l'ensemble de l'œuvre de Goethe se trouve être précisément le problème de la métamorphose d'une forme en une autre et le renouvellement du concept de forme qui y est associé : "nous résumons en lui un ensemble de relations toujours plus riches grâce auxquelles les éléments empiriques, auparavant séparés, s'ordonnent en séries, qui manifestent en elles-mêmes une configuration stable de leurs éléments aussi bien qu'une liaison et une subordination réciproques en fonctions de principes fixes ${ }^{66}$. Ce qui intéresse Cassirer dans la méthode morphologique, c'est la variation imaginative réglée qu'elle permet de développer, la dérivation créative à partir d'un point marquant du réel, efficace et fécond. Il décrit le rôle de l'imagination créatrice du poète comme une prolifération de formes, non pas suivant la fantaisie arbitraire, mais par des mises en série réglées dont le principe peut être indiqué en partant de cas saillants. Citons ici encore le passage commenté par Cassirer, extrait de l'écrit de Goethe intitulé "Das Sehen in subjektiver Hinsicht »: " J'avais ce don, quand je fermais les yeux, tête basse, pour me représenter une fleur au centre de mon organe de vue, que celle-ci ne se figeait pas un seul instant sous sa forme première mais elle s'ouvrait et dans son centre s'épanouissaient de nouvelles fleurs aux pétales colorés et feuilles vertes; ce n'étaient pas des fleurs naturelles, mais des fleurs imaginaires, bien que régulières comme les rosaces d'un sculpteur. Il était impossible de fixer cette création jaillissante, elle durait en revanche aussi longtemps que je le désirais, sans gagner ni perdre de sa force. Je pouvais de même, quand je me représentais un vitrail, faire naître son décor polychrome qui ensuite se transformait sans cesse depuis le centre jusqu'à la périphérie, à l'image du kaléidoscope récemment inventé ${ }^{67}$.

Sur un point précis toutefois, Cassirer modifie la conception goethéenne de la Gestalt, qu'il définit, dans une lettre à Warburg68, comme mise en figure dynamique. Et c'est précisément sur le rapport de la Gestalt aux mathématiques ${ }^{69}$. Alors que Goethe s'est explicitement opposé aux mathématiques de son temps, Cassirer, ayant étudié l'évolution des mathématiques proposée par le groupe d'Erlangen autour de Felix Klein $^{70}$, réinterprète la Gestalt goethéenne non seulement comme mise en série réglée à partir d'un cas saillant, un point du réel efficace et fécond, mais comme principe d'un groupe de transformation. Or, à partir de cette harmonisation de la Gestalt goethéenne et des dernières avancées mathématiques de son temps, Cassirer propose de la Gestalt une conception finalement très proche de celle que Lévi-Strauss va déployer pour élaborer une "science du concret $»^{71}$ autour de la notion de structure.

Il faut noter que Cassirer a très tôt conscience de l'importance de la linguistique développée par Roman Jakobson. Dès 1933, dans le Journal de Psychologie, il publie un article sur l'importance du langage dans la construction du monde de l'objet ${ }^{72}$. Or, LéviStrauss dit dans une lettre datée de 1971 à Werner Hoffman que cet article correspond « très exactement » à sa pensée, prétextant, visiblement quelque peu embarrassé par la question de Hoffman concernant la proximité de son système de pensée avec celui de Cassirer, que quelque chose de l'héritage cassirerien a pu lui parvenir via Jakobson ${ }^{73}$. Malgré le fait que Lévi-Strauss n'avoue pas connaître directement Cassirer, il faut toutefois souligner qu'il a dû nécessairement le croiser dans le Cercle Linguistique de 
New York, où ils publièrent tous deux aux côtés de Jakobson des articles décisifs sur le problème de l'application possible de la méthode structurale à l'ensemble des sciences humaines ${ }^{74}$. Cassirer est arrivé sur le bateau de l'exil le conduisant de Suède aux EtatsUnis dès 1941 avec Jakobson, tandis que Lévi-Strauss arrive quelques mois plus tard sur le Capitaine-Lemerle. Cassirer prend conscience qu'une nouvelle épistémè est en train de prendre forme autour de la notion de Gestalt. Dans un article sur « Le structuralisme dans la linguistique contemporaine " paru peu après sa mort en 1945 dans un des premiers numéros de la revue Word, il lit les symptômes de cette nouvelle vision du monde à la fois dans la psychologie de la forme de son cousin Kurt Goldstein, dans la linguistique structurale de Jakobson et dans la science de la forme de Wölfflin et nomme explicitement ce nouveau tournant langagier par l'appellation globale de "structuralisme», ce qu'il est le premier ou un des premiers à faire si explicitement ${ }^{75}$. L'originalité de son approche est cependant d'ancrer ce nouveau tournant langagier dans le tournant impulsé par la morphologie goethéenne et par la théorie de la langue de Humboldt ${ }^{76}$. Il faut alors souligner le rôle décisif qu'a joué Cassirer pour transformer la Gestalt goethéenne en structure, au sens que ce terme prendra bientôt avec LéviStrauss. Ce dernier reconnaît explicitement l'importance de la morphologie goethéenne, de la psychologie de la Gestalt, de la théorie de la langue de Humboldt et de Bühler ${ }^{77}$ et de la théorie des groupes de transformation de Félix Klein ${ }^{78}$. Qui d'autre que Cassirer a pu lui suggérer une telle synthèse? Il est curieux que Lévi-Strauss, choisissant après la fin de la guerre de rester encore quelques années à New York avec Jakobson, ait passé sous silence la philosophie des formes symboliques...

Il demeure toutefois qu'il faut admettre des divergences nettes entre l'approche que fait Cassirer de l'art et de la pensée mythique avec celle que propose Lévi-Strauss. Le spécialiste de Lévi-Strauss qu'est Gildas Salmon a suggéré habilement de parler d'une différence de «style» entre les deux auteurs ${ }^{79}$. C'est sur ce point que je souhaiterais montrer la proximité cette fois de Cassirer avec Warburg, qui n'est pas dénuée d'intérêt pour interroger le rapport du texte à l'image chez ces auteurs.

\section{La « symbolisation rituelle ${ }^{80}$ : le symbole comme acte impliquant le corps en mouvement qui perçoit}

27 Ayant travaillé de nombreuses années dans la Bibliothèque Warburg, Cassirer entretient en effet une forte proximité avec l'iconologie d'Aby Warburg, tandis que l'on pourrait dire que Lévi-Strauss va adopter dans son esthétique une conception plus panofskyienne de cette méthode. Il est à noter qu'il n'est pas anodin que Lévi-Strauss choisisse dans Regarder écouter lire de commenter l'approche panofskyenne de Poussin et le thème emblématique de son iconologie $E t$ in arcadia ego ${ }^{81}$. Sous la même appellation d'iconologie, se cache en effet deux conceptions très divergentes du rapport texte/ image. Alors que Lévi-Strauss et Panofsky vont établir leurs études sur le principe d'un primat du texte sur l'image, correspondant explicitement chez Lévi-Strauss à un primat du mythe sur le rituel ${ }^{82}$, Cassirer et Warburg vont, sur le modèle de l'ethnologie élaborée sur le terrain par Konrad Theodor Preuss, défendre explicitement un primat du rituel sur le mythe ${ }^{83}$. Dans une lettre de Warburg à Cassirer, il désigne leur projet commun: établir une science universelle de la culture comme anthropologie de l'homme en mouvement ${ }^{84}$. Warburg va avoir recours au modèle fournit par le linguiste Osthoff ${ }^{85}$ : ce dernier a observé que le changement de radical d'un verbe peut 
paradoxalement se produire sans modification du potentiel énergétique de ce verbe, et même avec une intensification remarquable de sa charge affective. Sur cette base, il devient possible de parler d'une grammaire des gestes et des affects, structurés sur la base des phénomènes de polarisation et de repolarisation produits par l'orientation et la réorientation du schéma corporel dans l'espace affectif construit dans les rituels et les productions artistiques. Or, à la lecture du second volume de Cassirer consacré dès 1925 à la pensée mythique comme espace de vie, de pensée et d'action, c'est bien de cette grammaire des gestes et de cette ritualisation symbolique qu'il s'agit ${ }^{86}$. La lecture de la riche correspondance entre Warburg et Cassirer témoigne largement des interactions et des points de convergence entre leur approche du symbole comme acte, comme oscillation entre l'Ergreiffen et le Begriff $f^{87}$.

Pour conclure, il faut toutefois souligner que dans ce que l'on a nommé les «Petites Mythologiques », et dans le "Finale » de L'homme nu, Lévi-Strauss a tenté de thématiser la conception de l'art comme "adhérence de la structure au sensible ${ }^{88}$ et donc de traduire en quelque sorte le phénomène de prégnance symbolique mis au jour par Cassirer et repris, comme on sait, par Merleau-Ponty dans sa Phénoménologie de la perception $^{89}$. Merleau-Ponty étant un grand ami de Lévi-Strauss, comme en témoigne la dédicace de La pensée sauvage au phénoménologue avec lequel il avait, aux côtés de Simone de Beauvoir, passé une année de stage d'agrégation de philosophie ${ }^{90}$. Mais la ligne de démarcation entre Cassirer et Warburg d'un côté ${ }^{91}$ et Lévi-Strauss et Panofsky de l'autre demeure : alors que pour les premiers le cœur de l'approche du symbole est le corps en mouvement qui perçoit et qu'ils cherchent à caractériser l'organisation d'un espace affectif, les seconds privilégient la dimension représentationnelle du langage et " négligent » pour ainsi dire, sauf ponctuellement, le corps et les affects. Cassirer et Warburg cherchent à définir une grammaire des gestes et à approcher le visuel sur le mode de l'organisation du rituel de la pensée mythique et construisent sur cette base la racine d'une anthropologie de l'homme en mouvement, tandis que Lévi-Strauss et Panofsky excluent la part affective de leur champ d'analyse et voient dans le rituel un " abâtardissement de la pensée » dont il faut, à en croire Lévi-Strauss, laisser l'étude à la seule biologie. Les premiers mettent au cœur de leur approche du réel le visuel organisé par le corps percevant, les seconds l'approche textuelle; ces quatre auteurs exploitant diversement et de manière contrastée, mais sans doute plus complémentaire qu'antagoniste, la richesse des rapports du texte à l'image et de l'image au texte.

\section{NOTES}

1. La référence au vocabulaire lévi-straussien pour qualifier la démarche d'E. Cassirer s'explicitera plus loin.

2. Pour une évaluation des positions de Croce par E. Cassirer, voir par exemple La philosophie des formes symboliques, tome I, Le langage, trad. O. Hansen-Love et J. Lacoste, Paris, Éditions de Minuit, 1972, abrégé PFS I, p. 125. Pour une comparaison des approches du langage et de l'esthétique de Cassirer et de Croce, voir Sarah Dessi Schmid, Ernst Cassirer und Benedetto Croce, 
Die Wiederentdeckung des Geistes, Ein Vergleich ihrer Sprachtheorien, trad. par R. Meisterfeld, Tübingen und Basel, A. Francke Verlag, 2005.

3. Silvia Ferretti, Cassirer, Panofsky and Warburg : Symbol, Art and History, traduit de l'italien en anglais par Richard Pierce, New Haven and London, Yale University Press, 1989, voir introduction, p. XIV et suivantes.

4. Michael Ann Holly, Panofsky and the Foundations of Art History, Ithaca and London, Cornell University Press, 1984, p. 108-109. Il y parle d'Aby Warburg, et le chapitre 5, p. 114, porte sur Panofsky et Cassirer.

5. Audrey Rieber, Art, histoire et signification - Un essai d'épistémologie d'histoire de l'art autour de l'iconologie d'Erwin Panofsky, Paris, L'Harmattan, 2012. Cet ouvrage permet de reconsidérer les approches des auteurs précédents, tout comme celle de G. Didi-Huberman, notamment celles que ce dernier défend dans L'image survivante. Histoire de l'art et temps des fantômes selon Aby Warburg, Paris, Éditions de Minuit, 2002, «Forces symptômales et formes symboliques : Warburg avec Cassirer ?», p. 433 et suivantes.

6. John Michael Krois, Bildkörper und Körperschema, édité par Horst Bredekamp et Marion Lauschke, Berlin, Akademie Verlag, 2011.

7. Ernst Cassirer, «Structuralism in Modern Linguistics » (1945), Word, Journal of the Linguistic Circle of New York, vol. 1, $\mathrm{N}^{\circ}$ 11, 1946 (August), réédité in Aufsätze und kleine Schriften [1941-1945], Hambourg, Felix Meiner Verlag, 2007, abrégé ECW 24. Voir Pierre Caussat, « Entre Humboldt et le structuralisme : la philosophie du langage d'Ernst Cassirer ", in E. Cassirer - De Marbourg à New-York, sous la direction de Jean Seidengart, Paris, Éditions du Cerf, 1990, p. 234 : "Il se pourrait - mais c'est là une hypothèse à confirmer - que le terme même de 'structuralisme' fasse ici sa première apparition publique; ce qui est sûr, à tout le moins, c'est que, rétrospectivement, ce texte lui ouvre droit de cité ou d'usage, indice de l'attention portée par son auteur aux mouvements qui renouvellent le travail de la pensée et reconnaissance du sens et des promesses qu'ils contiennent ».

8. Muriel van Vliet, « De la forme symbolique d'Ernst Cassirer à l'Anthropologie structurale de Claude Lévi-Strauss : portée et limites d'une comparaison », in Philosophie, numéro 115, Paris, Éditions de Minuit, automne 2012 (D. Pradelle, Dir.), Symbole et Société, p. 45-58.

9. Pour reprendre ici une expression de Gildas Salmon pour qualifier l'approche lévi-straussienne de la culture.

10. John M. Krois, «Die Universalität der Pathosformeln. Der Leib als Symbolmedium », in Quel Corps ? Eine Frage der Repräsentation, édité par H. Belting, D. Kamper et Martin Schulz, München, Wilhelm Fink Verlag, 2002, p. 295-307.

11. Idem, p. 87 : «Il n'existe pas de formule de Pathos sans insertion dans une narration, mais l'action dramatique des mythes ne peut, selon la théorie du mythe propre à Warburg et à Cassirer, provenir que des gestes qui les précèdent, c'est-à-dire d'un rituel, et pas l'inverse. Au début était l'action, non le mot. C'est là que réside la grande différence entre le sens que Warburg donne à la formule de pathos et celle que donne Lévi-Strauss aux masques indiens ", nous traduisons. J. M. Krois fait sur ce point des parallèles intéressants entre le pragmatisme (et la séméiologie) de C.S. Peirce et des positions conciliables avec le pragmatisme décelables chez Goethe et Cassirer.

12. Nous prenons donc ici, tout comme Audrey Rieber, une certaine distance avec les analyses de G. Didi-Huberman, qui ont eu toutefois le mérite de s'appuyer sur les textes fondamentaux de Cassirer, notamment ceux concernant la prégnance symbolique (PFS III) et de bien les mettre en lumière, ce qui n'avait pas été fait auparavant par les critiques français. L'approche de l'œuvre globale de Cassirer par G. Didi-Huberman reste cependant très lacunaire, ce qui le conduit ultimement à une réduction abusive de ses conceptions au seul néokantisme de l'École de Marbourg. 
13. Carlo Severi et Michael Houseman, Naven ou le donner à voir, Essai d'interprétation de l'action rituelle, Paris, CNRS Editions, Éditions de la maison des sciences de l'homme, 2009. Voir notamment la troisième partie, le chapitre 9, p. 219 et suivantes. L'introduction fait état de la difficulté d'approcher le rituel : soit on a approché le rituel en analysant ses significations, soit ses fonctions, mais sans analyse de "la qualité essentiellement agie des rituels ", les approches du rituel ne peuvent « rendre compte de leur organisation en tant que totalités obéissant à des principes de structuration internes». Les auteurs de cet ouvrage "centrent l'attention sur les implications relationnelles de la ritualisation» en référant le rituel au "réseau de relations actualisé au cours de la cérémonie » (p. 11).

14. Aby Warburg à Ernst Cassirer, lettre du 15 avril 1924, ECN 18, p. 67, nous traduisons : «(...) l'ellipse <sous-entendu le projet képlérien> est le point de départ ou encore la ligne de partage qui marque un changement de temps [Wetter, au sens de temps climatique], si nous voulons tous deux inaugurer 'une science générale de la culture comme théorie de l'homme en mouvement' ». 15. Muriel van Vliet, La forme selon Ernst Cassirer - De la morphologie au structuralisme, Rennes, Presses Universitaires de Rennes, 2013.

16. Fabien Capeillères, «Cassirer, Wölfflin, Panofsky - Sur la constitution de l'art comme forme symbolique ", in Cassirer et l'art comme forme symbolique, dirigé par M. van Vliet, Rennes, Presses Universitaires de Rennes, 2010.

17. André Stanguennec, « Néokantisme et hégélianisme chez E. Cassirer », in Ernst Cassirer - de Marbourg à New York, ouvrage collectif sous la direction de Jean Seidengart, Paris, Éditions du Cerf, 1990, p. 55-67. Dans l'introduction à cet ouvrage, Jean Seidengart parle de Cassirer comme «l'héritier de l'École de Marbourg » (p. 12).

18. Ernst Cassirer, Philosophie des formes symboliques, volume I, Le langage, traduit par Ole HansenLove et Jean Lacoste, Paris, Editions de Minuit, 1972, abrégé PFS I, chapitre 1, « Le problème du langage dans l'histoire de la philosophie », p. 61 et suivantes.

19. G.W. Hegel, Encyclopédie des sciences philosophiques, Philosophie de l'esprit, traduit par B. Bourgeois, Paris, Vrin, 1994, I. L'esprit subjectif, §458, Remarque, p. 253.

20. Ernst Cassirer, «Die kantische Elemente in Wilhelm von Humboldts Sprachphilosophie » (1923), Aufsätze und kleine Schriften, 1922-1926, Hambourg, Felix Meiner Verlag, 2003, abrégé ECW 16.

21. Voir sur ce point J. M. Krois, "More than a Linguistic Turn in Philosophy. The semiotic Programs of Peirce and Cassirer ", in Bildkörper und Körperschema, édité par H. Bredekamp et Mrion Lauschke, Berlin Akademie Verlag, 2011, p. 92 et suivantes.

22. A. Stanguennec, Être, soi, sens - Les antécédences herméneutiques de La dialectique réflexive, Villeneuve d'Ascq, Presses Univ. du Septentrion, 2008, p. 99-138.

23. Idem, p. 56.

24. PFS I, p. 24.

25. Ernst Cassirer, Problème de la connaissance dans la philosophie et la science des Temps Modernes, tome 3, Les Postkantiens, abrégé PCPS III, trad. par le Collège de France revue par C. Bouchindhomme, Paris, Éditions du Cerf, 1999, p. 308.

26. A. Stanguennec, op. cit., p. 57.

27. PCPS III, p. 288.

28. Ernst Cassirer, La philosophie des formes symboliques, tome II, La pensée mythique, traduit par Jean Lacoste, Paris, Editions de Minuit, 1972, abrégé PFS II.

29. PFS II, «Le mythe comme forme de pensée », p. 49 et suivantes, «Le mythe comme forme de vie (...) », p. 185 et suivantes.

30. E. Cassirer, La philosophie des formes symboliques, tome III, trad. par C. Fronty, Paris, Éditions de Minuit, 1972, p. 229.

31. PFS I, p. 130. Cassirer utilise l'édition anglaise suivante: Charles Darwin, The Expressions of the Emotions in Man and Animals, London, 1872. Voir le commentaire circonstancié qu'en donne 
Georges Didi-Huberman, L'image survivante, Histoire de l'art et temps des fantômes selon Aby Warburg, Paris, Éditions de Minuit, 2002, et notamment voir les illustrations qu'il choisit p. 228-229, p. 232-233 et p.239. Cf. notre contribution plus détaillée sur le rapport Cassirer/Darwin, "Grammaire des affects et phénomène d'expression selon A. Warburg et E. Cassirer ", in Langage et affectivité, ouvrage collectif dirigé par Samuel Le Quitte et Gabriel Mahéo, Paris, Le cercle herméneutique, 2014.

32. PFS I, p. 127 et suivantes.

33. PFS I, p. 128.

34. PFS I, p. 129.

35. Idem.

36. Idem.

37. G. W. F. Hegel, Phénoménologie de l'esprit, trad. par B. Bourgeois, Paris, Vrin, 2006, p. 131 : « La certitude sensible ou : le ceci et la visée comme telle mienne ».

38. PFS II, p. 304-305.

39. Il s'agit ici d'une convention progressivement adoptée par les traducteurs de Cassirer pour différencier Vorstellung (représentation, qui suppose une mise à distance, " intellectualisante ») et Darstellung (présentation, articulation d'un signifiant et d'un signifié, sans impliquer nécessairement encore une position intellectualisante « de surplomb»). La Darstellung est une sorte de seuil (ou de pli). Selon Cassirer, il ne peut y avoir de Vorstellung sans Darstellung, c'està-dire sans présentification dans un matériau sensible et, réciproquement, il ne peut y avoir conscience et mise en lumière de la Darstellung sans Vorstellung. On pourrait traduire aussi Darstellung par présentification, incorporation, incarnation.

40. Ernst Cassirer, Gesammelte Werke, Hamburger Ausgabe, Band 16, Aufsätze und kleine Schriften (1922-1926), édité par Birgit Recki, Hamburg, Meiner Verlag, 2003, abrégé ECW 16, p. 311 : « Il y a un domaine de l'esprit dans lequel le mot ne conserve pas seulement sa force d'image originaire, mais à l'intérieur de celle-ci la rénove constamment ; dans laquelle il fait l'expérience en une certaine mesure de sa palingénésie constante, de sa renaissance tout à la fois sensible et spirituelle. Cette régénération s'accomplit en tant qu'il se transforme en expression artistique. Il a ici à nouveau en partage la plénitude de la vie : cette vie n'est plus la vie contrainte du mythe, mais la vie libérée de l'esthétique ». Notre traduction, nous soulignons.

41. PFS II, p. 305.

42. PFS I, p. 132: "C'est un passage constant qui s'établit de la 'préhension' (Ergreiffen) à la 'compréhension' (Begriff)». Warburg reprend cette oscillation entre l'Ergreiffen et le Begriff pour qualifier le symbole, cf. «Introduction à l'Atlas Mnémosyne », in Aby Warburg, Miroirs de faille - à Rome avec G. Bruno et É. Manet, 1928-1929, Paris, Presses du réel, 2011, p. 140 : « Entre l'imagination qui s'empare de l'objet et la pensée conceptuelle qui le contemple à distance, se situe ce qu'on appelle l'acte artistique, qui n'est autre qu'une manipulation tactile de l'objet aboutissant à son reflet plastique ou pictural ».

43. E. Cassirer, « Sprache und Mythos - Ein Beitrag zum Problem der Göttername », in ECW 16.

44. PFS II, p. 61 : «Ce n'est pas un simple spectacle, une simple pièce, qu'exécute le danseur qui participe à un drame mythique : le danseur est le dieu, il devient le dieu. Tous les rituels de la végétation en particulier, dans lesquels on célèbre la mort et la résurrection du dieu, expriment ce sentiment essentiel de l'identité, cette identification réelle. Ce qui a lieu dans ces rites, comme dans la plupart des rites mystérieux, n'est pas une simple mise en scène qui imiterait un événement, mais l'événement lui-même, son accomplissement immédiat : c'est un drômenon, un événement réel et effectif, parce que parfaitement efficace ».

45. PFS II, p. 304-305.

46. G. Simondon parlera de manière similaire sur ce point avec Cassirer de "phases ", un terme qui pourrait bien traduire «Urteilung » chez Cassirer.

47. ECW 16, p. 302.

Images Re-vues, Hors-série 5 | 2016 
48. Dans son ouvrage intitulé Manières de faire des mondes, trad. par M.-F. Popelard, Folio Essais, 2006, Nelson Goodman se réfère explicitement, bien qu'indirectement et avec des déplacements notables, à Cassirer.

La terminologie lévi-straussienne («homologies structurales») est employée ici à dessein, comme nous le justifierons plus loin.

49. On pourrait qualifier ce réseau de système « complexe ", comme on voit, par exemple, avec certaines similitudes, le spécialiste des techniques que fut Simondon en constituer un.

50. Pour reprendre la thèse de Birgit Recki, Kultur als Praxis. Eine Einführung in Ernst Cassirers Philosophie der symbolischen Formen, Berlin, 2004.

51. E. Panofsky thématise les trois niveaux de signification caractéristiques de l'iconologie dans trois textes : Essais d'iconologie, Introduction, p. 13 et suivantes, notamment tableau p. 31, L'œuvre d'art et ses significations, p. 27 et La perspective comme forme symbolique, "La contribution au problème de la description des œuvres ", p. 235, et notamment le tableau, p. 255.

52. Panofsky, Essais d'iconologie, chapitre IV, «L'amour aveugle», p. 151 et suivantes. Voir illustration 106.

53. Il faut nuancer cet avis toutefois, si l'on considère l'intérêt pris aux Andachtsbilder, ou images de la dévotion in Erwin Panofsky, Peinture et dévotion en Europe du Nord à la fin du Moyen Age, présentation par D. Arasse, Paris, collection « Idées et recherches », Flammarion, 1997.

54. Le recours à une expression caractéristique de M. Foucault se justifie pleinement ici si l'on a en tête le souci propre à Cassirer et Panofsky de mettre au jour des «aires relationnelles » où se projettent "divers langages» religieux, économiques, artistiques... (selon une expression d'E. Cassirer, Liberté et forme, L'idée de la culture allemande, CEuvres XLV, trad. par J.Carro, M. Willemann-Carro et J. Gaubert, Paris, Éditions du Cerf, 2001, p. 9). Foucault fait l'éloge de Cassirer dans sa recension de La Philosophie des Lumières (premier texte de Cassirer traduit en français chez Fayard, par Pierre Quillet) dans La Quinzaine Littéraire, n8, 1er-15 juillet, en 1966 : "Une histoire restée muette "; l'année où paraît son ouvrage majeur Les Mots et les Choses, Paris, Gallimard, 1966, qui se réfère explicitement à la philosophie des formes symboliques, p. 358 : « là peuvent apparaitre et sont en effet apparues les diverses philosophies de la vie, de l'homme aliéné, des formes symboliques ». Pour une comparaison de l'histoire de l'épistémologie des sciences humaines chez Cassirer et Foucault, voir Muriel van Vliet, "L'histoire de l'art: un paradigme pour penser la logique des sciences de la culture ? ", Revue Appareil, n 9-2012, http:// revues.mshparisnord.org/appareil/index.php/pdf/index.php?id=1435.

55. E. Panofsky, Architecture gothique et pensée scolastique, traduction et postface de Pierre Bourdieu, Éditions de Minuit, Paris, 1967, chap. 2, « force formatrice d'habitudes », p. 83.

56. E. Panofsky, Essais d'iconologie, trad. par C. Herbette et B. Teyssèdre, Paris, Gallimard, 1967, p. 21 : «Nous envisageons l'œuvre d'art en tant que symptôme de quelque 'autre chose', qui s'exprime en une infinie diversité d'autres symptômes; et nous interprétons les caractères de sa composition et de son iconographie en tant que manifestations plus particulières de cette 'autre chose'. La découverte et l'interprétation de ces valeurs symboliques (en général ignorées de l'artiste, parfois même fort différentes de ce qu'il se proposait consciemment d'exprimer) est l'objet de ce que nous pouvons appeler iconographie au sens large : une méthode d'interprétation qui procède d'une synthèse plutôt que d'une analyse ». Nous soulignons.

57. E. Panofsky, La perspective comme forme symbolique, trad. G. Ballangé, Paris, Éditions de Minuit, 1975.

58. Voir la postface de Pierre Bourdieu, à E. Panofsky, Architecture gothique et pensée scolastique, où le sociologue explique d'où il a tiré l'expression d'habitus culturel, en lui donnant toutefois une inflexion nouvelle, p. 151. La question de l'orientation spatiale (symbolique) est au cœur de ses réflexions sur l'habitat kabyle.

59. J. M. Krois, "Urworte : Cassirer als Goethe-Interpret », in E. Rudolph/Küppers, Bernd-Olaf (éd.), Kulturkritik nach Ernst Cassirer, Cassirer-Forschungen, Band 1, Hambourg, Meiner Verlag, 
1995, p. 297-324 ; «Die Goetheschen Elemente in Cassirers Philosophie », in B. Naumann/ B. Recki., Cassirer und Goethe, Neue Aspekte einer philosophisch-literarischen Wahlverwandtschaft, Berlin, Akademie Verlag, 2002, p. 157-172.

60. E. Cassirer, Liberté et forme, L'Idée de la culture allemande, CEuvres XLV, trad. de Jean Carro, Martha Willman-Carro et Joël Gaubert, Paris, Éditions du Cerf, 2001.

61. Idem, p. 261 : «Il est au fond parfaitement oiseux de discuter le nombre de traits de caractères de Méphisto qui rappellent Merck ou Herder, ou de se demander si dans le personnage de Gretchen est exclusivement conservée l'image de Friederike ou si des souvenirs des années d'enfance ou d'adolescence y prennent une part active. En effet, de cette manière on n'approchera pas le moins du monde le véritable rapport qui existait pour Goethe entre la vie et l'art. Ce n'est pas l'existence de Goethe dans son contenu, mais ses principes formels dans leur devenir et leur variation qui sont, involontairement, représentés dans le cycle de Faust ». Nous soulignons.

62. Dans ECW 16, édité par B. Recki, texte établi et annoté par Julia Clemens, Hambourg, Meiner Verlag, 2003, figure l'essai « Kant und Goethe » (1924). Dans ECW 14, édité par Birgit Recki, texte établi et annoté par R. Becker, Hambourg, Felix Meiner Verlag, 2004, figurent notamment : " Goethe Idee der Bildung und Erziehung ", "Goethe und die geschichtliche Welt ", " Goethe und das 18. Jahrhundert », « Goethe und Plato »...

63. E. Cassirer, Liberté et forme, p. 188.

64. Claude Lévi-Strauss, Mythologiques, tome 4, L'homme nu, p. 561.

65. Liberté et forme, p. 212.

66. Liberté et forme, p. 217. Cassirer cite comme élément central de l'œuvre de Goethe le poème : « Metamorphose der Thiere ", in Werke, 1. Abt., Bd. III, S. 89 ff, s. 91.

67. Ernst Cassirer, Liberté et forme, p. 233 : «J'avais ce don, quand je fermais les yeux, tête basse, pour me représenter une fleur au centre de mon organe de vue, que celle-ci ne se figeait pas un seul instant sous sa forme première mais elle s'ouvrait et dans son centre s'épanouissaient de nouvelles fleurs aux pétales colorés et feuilles vertes; ce n'étaient pas des fleurs naturelles, mais des fleurs imaginaires, bien que régulières comme les rosaces d'un sculpteur. Il était impossible de fixer cette création jaillissante, elle durait en revanche aussi longtemps que je le désirais, sans gagner ni perdre de sa force. Je pouvais de même, quand je me représentais un vitrail, faire naître son décor polychrome qui ensuite se transformait sans cesse depuis le centre jusqu'à la périphérie, à l'image du kaléidoscope récemment inventé ».

68. E. Cassirer à A. Warburg, 12 avril 1924, in ECW 18, p. 65 : «Si nous voulons introduire une morphologie, nous ne devrions jamais parler de figure (Gestalt), ou, si nous faisons usage de ce mot, nous devrions en tout cas avoir à l'esprit une idée, un concept, certes, mais enveloppé dans l'expérience et dont la fermeté n'est saisissable que dans l'espace d'un instant ».

69. E. Cassirer, Idee und Gestalt, Darmstadt, Wissenschaftliche Buchgesellschaft, 1971, "Goethe und die mathematische Physik. Eine erkenntnistheoretische Betrachtung ", p. 33 et suivantes.

70. Ernst Cassirer, "The concept of group and the theory of perception " [1944], in ECW 24, Aufsätze und kleine Schriften 1941-1946, Hambourg, Felix Meiner Verlag, 2007. Cassirer envisage d'appliquer le concept de groupe tel que le développe F. Klein à des champs autres que les mathématiques, notamment à la perception.

71. C. Lévi-Strauss, La pensée sauvage, Paris, Plon, 1962, chap. premier, «La science du concret », p. 11 et suivantes.

72. Ernst Cassirer, «Le langage et la construction du monde des objets ", édité par P. Guillaume, Journal de Psychologie Normale et Pathologique, vol. XXX, 1933, pp. 18-44. Réédité dans Psychologie du langage par H. Delacroix, E. Cassirer, etc., Bibliothèque de Philosophie contemporaine, Paris, Alcan, 1933 et dans Essais sur le langage, édité par J.-C. Pariente, Paris, Minuit, 1969. Paru à l'origine en allemand : «Die Sprache und der Aufbau der Gegenstandswelt », Bericht über den XII. Kongress der deutschen Gesellschaft für Psychologie, Hambourg, Jena, édité par G. Fischer, 1932. 
73. Werner Hofmann, "Meine Wege zu Cassirer, eine Skizze", in Theory of Figuration, Cassirerstudies II-2009, Naples, Bibliopolis, pp. 54-63 : Lévi-Strauss lui aurait écrit dans une lettre datée du 25 octobre 1971 : «J'ai très peu fréquenté l'œuvre de Cassirer et je ne crois pas avoir été influencé par elle. Mais il y a deux ans environ, j'ai pris connaissance d'un article de lui, publié en français dans le 'Journal de psychologie', si je ne me trompe, en 1933, qui m'a paru correspondre très exactement à ma propre pensée. Il est d'ailleurs possible que quelque chose de l'enseignement de Cassirer me soit parvenu, sans que je le sache, par l'intermédiaire de Roman Jakobson qui, je crois, le connaissait personnellement ».

74. Tandis qu'E. Cassirer y publia l'article sur le structuralisme ci-dessus mentionné, Lévi-Strauss y publia ce qui deviendra le second chapitre de l'Anthropologie structurale (Paris, Plon, 1974, p. 43 et suivantes), "L'analyse structurale en linguistique et en anthropologie », publié sous ce titre dans : Word, Journal of the Linguistic Circle of New York, vol. 1, n², August 1945, p. 1-21.

75. ECW 24, p. 320.

76. ECW 24, p. 315.

77. C. Lévi-Strauss, Anthropologie structurale, op. cit., p. 380-381: «Plus profondément enfin, un sociologue norvégien, M. Sverre Holm, après avoir lui aussi remarqué que «la science de la culture " s'est, depuis longtemps, inspirée du message de la Gestalt-Psychologie », essaye de rattacher directement le structuralisme à l'une des sources lointaines de la pensée gestaltiste, la philosophie naturelle de Goethe. Quant au linguistes structuralistes, Troubetzkoy et Jakobson ont souvent reconnu leur dette envers la Gestalt, notamment aux travaux de K. Bühler ». Goldstein est souvent mentionné, comme par ex., p. 343. Pour la référence à Goethe, voir Les Mythologiques, L'homme nu, p. 604 et 620, où Lévi-Strauss cite le même passage de Goethe que Cassirer dans la revue Word.

78. Pour la référence à Félix Klein, voir notamment Les Mythologiques, L'homme nu, p. 188, 240, 243-244, 289, 581-582.

79. Conférence prononcée lors du colloque «E. Cassirer et l'exil américain (194-1945) », organisé par Sabine Plaud et moi-même à l'ENS les 16 et 17 novembre 2012, disponible sur le site du groupe de recherche Transfers (J. Benoist).

80. Voir référence à l'ouvrage de M. Houseman et C. Severi mentionné ci-dessus.

81. C. Lévi-Strauss, Regarder écouter lire, Paris, Plon, 1993, p. 19.

82. C. Lévi-Strauss, Les Mythologiques, L'homme nu, p. 603. Le rituel y est présenté comme un «abâtardissement de la pensée ». Cité par J. M. Krois, Bildkörper und Körperschema, p. 87.

83. E. Cassirer, PFS II, p. 61 : la pensée mythique se manifeste non dans les mythes écrits, mais dans le drômenon, la procession, la cérémonie rituelle. Voir aussi p. 187: "Le monde de la représentation mythique se révèle par conséquent très étroitement lié au monde de l'agir, et cela dans ses formes primitives les plus immédiates ».

84. Lettre citée précédemment.

85. Aby Warburg, Miroirs de faille, op. cit., p. 141 : «cette étude <de Osthoff> démontrait en substance qu'un changement de radical pouvait s'opérer dans certaines formes de verbes conjugués et le comparatif de certains adjectifs sans affecter l'identité énergétique de la qualité ou de l'action signifiée, lors même qu'avait disparu l'identité formelle de l'expression lexicale de base. Au contraire, l'apparition d'une expression portant un radical nouveau produisait même une intensification de la signification première ".

86. PFS II, p. 122 : «(...) la pensée tout entière, comme la perception et l'intuition sensibles, reposent sur une base affective originaire. Aussi loin que pourront aller la subtilité et les articulations de sa structure, l'espace mythique restera, dans sa totalité, enfoncé dans cette base, et pour ainsi dire enseveli. Si nous parvenons à poser dans cet espace certaines limites et certaines distinctions, ce n'est donc pas par une détermination progressive de la pensée, par les voies de l'analyse et de la synthèse intellectuelle. Les différenciations de l'espace renvoient finalement aux différenciations qui s'effectuent dans cette base affective même ». 
87. Voir ECW 18, p. 57, Lettre de Cassirer à F. Saxl, parlant de Warburg: «Je crois que le moyen terme qu'il cherche entre le symbole mythique et le 'concept' abstrait, entre le Mythos et le Logos, doit avant tout être recherché dans la forme spirituelle spécifique du langage, qui est toujours à la fois vivification et détermination, personnification et objectivation ", nous traduisons. Comparer sur ce thème PFS I, p. 132: «C'est un passage constant qui s'établit de la 'préhension' (Ergreiffen) à la 'compréhension' (Begriff)» et A. Warburg, Miroirs de faille, p. 140 : «Entre l'imagination qui s'empare de l'objet et la pensée conceptuelle qui le contemple à distance, se situe ce qu'on appelle l'acte artistique (...)».

88. Mythologiques, L'homme nu, op. cit., p. 578, 580, pour ne donner que deux exemples centraux.

89. Voir Muriel van Vliet, «Phénoménologie de la perception et théorie de l'art chez E. Cassirer : l'ouverture d'un dialogue avec M. Merleau-Ponty", in Cassirer et l'art comme forme symbolique, dirigé par M. van Vliet, Rennes, Presses Universitaires de Rennes, 2010, p. 117 et suivantes.

90. C. Lévi-Strauss, La pensée sauvage, op. cit., Préface : "Ceux qui nous ont approchés, MerleauPonty et moi, au cours des récentes années, connaissent quelques-unes des raisons pour lesquelles il allait de soi que ce livre, qui développe librement certains thèmes de mon enseignement au Collège de France, lui fût dédié. Il l'eût été de toute façon s'il avait vécu, comme la continuation d'un dialogue dont le début remonte à 1930, quand, en compagnie de S. de Beauvoir, nous nous sommes rencontrés à l'occasion d'un stage pédagogique à la veille de l'agrégation ».

91. Et, pourrions-nous ajouter, Merleau-Ponty.

\section{RÉSUMÉS}

Cet article porte sur le rapport complexe de l'art au langage chez le philosophe allemand Ernst Cassirer (1874-1945) et cela selon trois niveaux distincts de compréhension de son œuvre. L'analyse de l'interprétation cassirerienne du tournant langagier qu'imprima le théoricien du langage Wilhelm von Humboldt au criticisme kantien permet, à un premier niveau, de mieux cerner le rapport de la philosophie des formes symboliques de Cassirer à l'iconologie d'Erwin Panofsky. Mais un second tournant langagier se joue encore chez Cassirer, lui permettant d'approfondir la conception morphologique de la Gestalt propre à Goethe dans la direction du structuralisme naissant, ce qui pourrait fort bien avoir été une des sources de Claude LéviStrauss. À un troisième et dernier niveau, cet article propose toutefois d'évaluer non seulement les points de convergence entre l'esthétique de Cassirer et celle du célèbre anthropologue français, mais aussi leurs points de divergence potentielle. Si le symbole est avant tout pour Cassirer un acte impliquant le corps en mouvement qui perçoit et si tout le processus de création du sens s'enracine chez lui dans un processus de symbolisation rituelle, Cassirer se positionnerait plutôt du côté de l'historien de l'art que fut Aby Warburg, tandis que Lévi-Strauss se rapprocherait davantage de Panofsky, dès lors qu'il s'agit de démêler les rapports qu'entretiennent art et langage.

\section{INDEX}

Mots-clés : Goethe, Cassirer, Panofsky, Warburg, Humboldt, Lévi-Strauss, morphologie, structuralisme, forme symbolique, iconologie, art, langage, sémiologie, symbolisation rituelle 


\section{AUTEUR}

\section{MURIEL VAN VLIET}

Muriel van Vliet est membre du CEPA (PhiCo-ExeCo, Paris 1), membre associé de l'équipe

«Philosophie des normes » (Rennes 1) et participe au groupe de travail « Morphologies » (dirigé par D. Cohn). Normalienne et agrégée de philosophie, elle a soutenu en juin 2011 une thèse sous la direction de C. Colliot-Thélène et de J. A. Barash, récemment parue sous le titre : La forme selon E. Cassirer - De la morphologie au structuralisme (Presses Universitaires de Rennes, 2013). Elle a également dirigé l'ouvrage collectif Cassirer et l'art comme forme symbolique (PUR, 2010) et publié des articles permettant de mesurer l'importance de la lecture de Cassirer par M. Merleau-Ponty, M. Foucault et C. Lévi-Strauss, dont « De la Philosophie des formes symboliques de Cassirer à l'Anthropologie structurale de Claude Lévi-Strauss : portée et limites d'une comparaison », dans la revue Philosophie (dirigée par D. Pradelle, numéro 115, automne 2012). Ses recherches récentes portent sur Georg Simmel et sur Edgar Wind. 